\title{
A Seroprevalence Study for HBV in Pregnant Women in Greece: High Risk Migrant Groups and Opportunities for Intervention
}

\begin{abstract}
Anagnostatou $\mathbf{N}^{1}$, Galanakis $\mathrm{E}^{2}$ and Hatzidaki $\mathrm{E}^{1 *}$ ${ }^{1}$ Department of Neonatology and NICU, University Hospital of Heraklion, University of Crete, Greece ${ }^{2}$ Department of Pediatrics, University Hospital of Heraklion, University of Crete, Greece

*Corresponding author: Hatzidaki E, Department of Neonatology and NICU, University Hospital of Heraklion, University of Crete, 7 Hatzispyrou Str. 71304, Heraklion, Crete, Greece
\end{abstract}

Received: March 30, 2021; Accepted: April 24, 2021; Published: May 01, 2021

\begin{abstract}
Background: Perinatal transmission of HBV leads to chronic infection in up to $90 \%$ of neonates. Focused prenatal screening and appropriate treatment of pregnant women and neonates is necessary for the elimination of hepatitis B, as was stated in the 2017 WHO Action Plan for HBV. Information on seroprevalence of HBV in Greece, especially in pregnant women, is scarce and rather outdated. Seroprevalence data specifically for high-risk groups, such as immigrants, is necessary for proper public health planning and elimination of vertical transmission and this study will struggle to fill the gap that exists in Greece.
\end{abstract}

Methods: HBsAg status of pregnant women delivering during 2017 in Crete was studied. Seroprevalence was estimated for the whole population and each ethnic group separately.

Results: The mean age of the participants was $30.38( \pm 6)$ years. Their origin was Greek (76.76 \%), Albanian (10.18\%), Bulgarian (3.79\%), Roma population (2.44\%), Russia and Former Republics of Russia (2.06\%), Romanian (1.95\%), Central Europe (0.70\%) refugees from Syria, Morocco, Egypt (0.55\%), and East Asian $(0.43 \%)$. The HBsAg (+) seroprevalence was $1.5 \%$. The seroprevalence of Greeks was $0.5 \%$, while Albanians, Bulgarians, Romanians and Roma had $4.3 \%, 5.7 \%, 2.8 \%$, and $11.1 \%$ respectively $(p<0.001)$.

Conclusions: Defining specific at-risk groups in each country is fundamental, since MTCT is the principal mode of transmission in high prevalence settings. Our study revealed high seroprevalence in certain migrant groups and Roma women. This information is essential for proper planning of perinatal care in Greece, especially taking into account that these underprivileged groups often lack quality health care.

Keywords: Perinatal infection; Mother-to-child-transmission; Seroprevalence; Pregnant women; Hepatitis B virus (HBV); Greece

\section{Abbreviations}

MTCT: Mother-to-Child-Transmission; CHB: Chronic Hepatitis B; HBV: Hepatitis B Virus

\section{Introduction}

Hepatitis B virus infection is a major health problem with an estimated 240 million chronic carriers worldwide and over 600,000 deaths per year. WHO has developed a global strategy to eliminate hepatitis B as a public health threat by 2030 , with a goal to reduce its incidence by $90 \%$ and its mortality by $65 \%$ [1]. Combating Mother-to Child-Transmission (MTCT) is highlighted as an intervention with great impact, since up to $90 \%$ of infected neonates become chronic carriers and account for almost $50 \%$ of chronic cases worldwide. Especially in settings with high hepatitis B prevalence, MTCT is a major mode of transmission, along with early childhood household infection of unvaccinated children. Accordingly, WHO has advocated universal immunization with a birth-dose $\mathrm{HBV}$ vaccine and universal HBsAg screening of pregnant women [1-4].
The main route of MTCT is vertical transmission during childbirth, by the HBV-infected body fluids or maternal blood entering fetal circulation during uterine contractions [5-8]. Intrauterine infection takes place in $13-44 \%$ of cases by placental barrier disruption or even genetic vertical infection of the fetus from HBV-infected oocytes or sperm [5,6], and horizontal infection through daily contact or breastfeeding also occurs, but to a lesser extent, putting unvaccinated children of HBV-infected mothers in danger of acquiring HBV infection until their 5th birthday [9]. The risk for perinatal transmission of $\mathrm{HBV}$ is $70-90 \%$ for infants born to mothers who are both HBsAg and HbeAg (+), compared to 5-20\% for infants born to HbeAg (-) mothers. The joint HBV standard passive-active immunopropylaxis with HBIG plus HBV vaccine in neonates within 12 hours after delivery is very effective at reducing HBV transmission to $5-9 \%$. The remaining percentage is mainly attributed to intrauterine infection, usually encountered with a high viral load and/or HBeAg positivity [5-8]. Wen WH et al, found that pregnant women with a high viral load, who mostly were also HBeAg $(+)$, presented with a risk of immunoprophylaxis failure ranging from 
$6.6 \%$ to $27.7 \%$ with an HBV load of 107 to 109 copies respectively [10]. HBeAg (+) pregnant women or those with a high viral load, are now considered for treatment with nucleotide analogs [5-7]. A systematic review and meta-analysis on the efficacy and safety of peripartum antiviral prophylaxis was recently undertaken and found all three antivirals (lamivudine, tenofovir disoproxil fumarate and telbivudine) highly efficious and safe [11]. Due to high barrier to resistance of tenofovir, the latest WHO recommendations suggest administering tenofovir to pregnant women infected with HBV with a high viral load from week 28 of pregnancy until at least childbirth to prevent MTCT. In settings in which antenatal HBV DNA testing is not available, $\mathrm{HBeAg}$ testing can be used as an alternative to determine eligibility for tenofovir prophylaxis [12].

With an estimated prevalence of 0.7-8.7 \% for hepatitis B infection in pregnant women in different parts of the world, a great number of neonates are in danger of perinatal transmission. Taking into account that less than $5 \%$ of $\mathrm{HBV}$ chronic carriers are aware of their status and that prenatal risk factor-based screening will miss many chronic HBV infections among pregnant women, screening for HbsAg during pregnancy should be universal; women at high risk should be offered repeat screening at delivery [7,11-13]. Additionally, in $\mathrm{HbsAg}(+)$ pregnant women $\mathrm{HBV}$ viral load, or at least $\mathrm{HBeAg}$, should also be tested [7].

Greece is considered a country of intermediate endimicity in the latest Technical report of ECDC on Epidemiological assessment of Hepatitis B among migrants in the EU, with an estimated HBsAg prevalence of 2.33 (CI 1.54-3.11), reflecting around 260.000 chronic HBV carriers. Even though only $6.4 \%$ of the population is foreignborn, $84 \%$ are from intermediate or high endimicity countries, thus contributing significantly to the HBV burden of Greece [14]. Data on HBV infection in pregnant women in Greece are scarce and rather outdated, with different studies reporting a prevalence of 1.2-3.8 \% [15]. According to WHO latest strategy, every country should define specific populations within their borders that are most at risk for HBV infection and response should be based on epidemiological and social context [1]. The goal of this study is to provide useful information on the prevalence of HBV infection in pregnant women residing in Greece, shedding light especially on vulnerable population groups such as migrants, which are at greatest risk of limited medical prenatal care.

\section{Patients and Methods}

\section{Study population}

All of the pregnant women who gave birth in the Obstetric Clinics of the three major public hospitals of Crete, Greece, (University Hospital of Heraklion, Venizeleio General Hospital of Heraklion and General Hospital of Chania) from January 2017 to December 2017 were included. Preliminary results from a similar survey that took place during December 2016 at the University Hospital of Heraklion were also included. Women experiencing miscarriage or stillbirth were excluded from the survey. After getting permission from the Ethic Committees of each hospital and the Directors of the individual clinics, we reviewed the medical records for prenatal HBV serological testing. In women with no prenatal screening documented, testing with HBsAg was ordered on an emergency basis. Records of women who were HBsAg (+) were further checked, to verify whether appropriate immunoprophylaxis against HBV had been offered to the newborn. Data that was also collected from the medical records included the ethnic origin of the participating women and their age.

Mean seroprevalence was calculated for the total of the study sample and then further analyzed according to the nationality of the participants. Comparisons were made among the different nationality groups, with previous studies, as well as with studies from the countries of origin.

\section{Statistical analysis}

An excel database was created initially for each hospital and at the end collectively for the whole study population. Data analysis was performed using Vassarstats and GraphPrism software. Specific prevalence was calculated in each ethnic group of pregnant women, and compared to the mean seroprevalence and that of the Greek women. Chi-square test was used to compare qualitative values, whenever appropriate. P-values $<0.05$ were considered statistically significant.

\section{Results}

Our study included a total of 2438 women who gave birth from January 2017 to December of 2017 in Crete. Results on HBV seroprevalence were available for 1846 women (75.7\%). The remainder records were either not found or specific data concerning HBV infection was missing, in which case it was postulated that serological tests had been done on a private basis and presented in printed form to the obstetrician, but not recorded in the medical file. In any case, these missing results were most possibly negative, since HBsAg positivity would have most probably been recorded. Women with no prenatal screening were always tested immediately postnatally. Negative prenatal tests for HBV were usually not repeated in the third trimester or at birth, even for women at high risk.

The mean age of the participants was $30.38( \pm 6)$ years. Three point 5 percent of women were $<20$ years old, $39.3 \% 20-29$ years old, $50.0 \%$ 30-39 years old, and $7.1 \%$ over 40 years old. Their origin was Greek (76.76\%), Albanian (10.18\%), Bulgarian (3.79\%), Roma population (2.44\%), from Russia and the former Republics of Russia (2.06\%), Romanian (1.95\%), from Central/Western Europe (0.70\%), refugees from Syria, Morocco, Egypt (0.55\%), and East Asian (0.43\%).

The seroprevalence rate of the study population for $\mathrm{HBsAg}$ (+) was $28 / 1846$ (1.5\%, 95\% CI 1-2.2). The highest seroprevalence of HbsAg was in women of Far East origin (37.5\%, 95\% CI 10-74) and in Roma women (11.1\%, 95\% CI 4.8-23.5). Bulgarian women had a seroprevalence of 5.7\% (95\% CI 2.2-14), Albanian 4.3\% (95\% CI 2-8.5) and Romanian 2.8\% (95\% CI 0.5-14) (Figure 1). There were no HbsAg (+) women from Russia and the former Republic of Russia, Central and Northern Europe and refugee group, but the number tested was small, so safe conclusions cannot be made. Greek women showed a low seroprevalence of $0.5 \%$ (95\% CI $0.2-1)$, and the difference compared to the mean seroprevalence of the whole population was statistically significant $(\mathrm{p}<0.01)$.

Among the seropositive women, 28.6\% were Albanian, 25\% Greek, 17.85\% Roma, 14.2\% Bulgarian, 10.7\% from Far East, and $3.6 \%$ Romanian (Figure 2). The women who tested positive for HBsAg were stratified according to age: there was no case under 20 years old, $32.14 \%$ were between $20-29$ years old, $60.71 \%$ between 30 - 


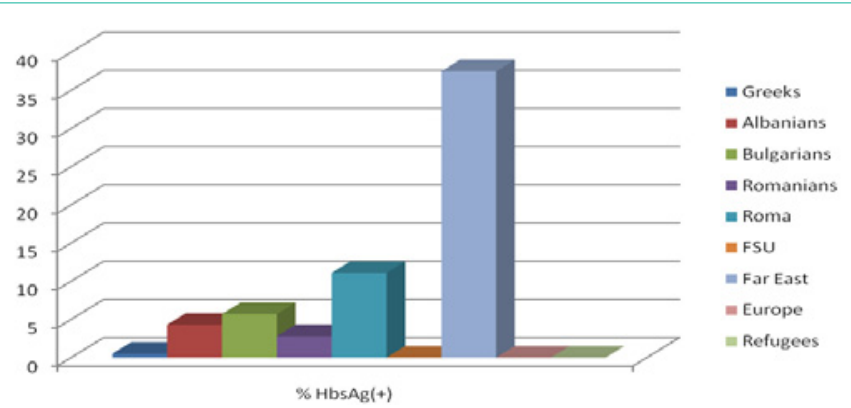

Figure 1: Seroprevalence of HBsAg (+) among different ethnic groups.

\section{Ethnic origin of $\mathrm{HBsAg(+)}$ pregnant women}

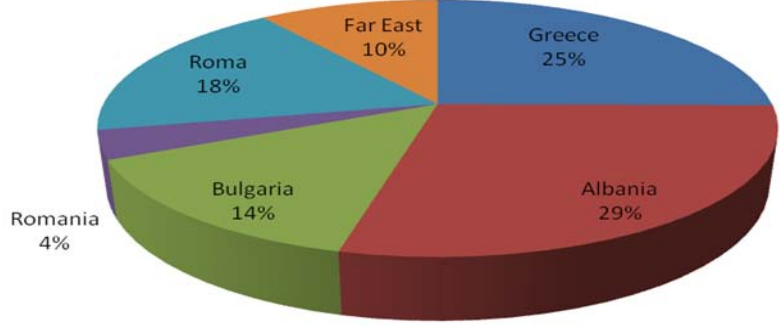

Figure 2: Ethnic origin of $\mathrm{HBsAg}(+)$ pregnant women.

39 years old and only $7.14 \%$ above 40 years old. This difference was statistically significant $(\mathrm{p}<0.001)$.

According to the medical records, appropriate passive and active immunoprophylaxis was given to all 28 newborns whose mothers were HBsAg $(+)$. Interestingly, in the medical records of HBsAg $(+)$ women, HbeAg and HBV viral load were not documented and there was no evidence that nucleotide analogs had been offered as treatment. Accordingly, calculations on the seroprevalence of HbeAg $(+)$ women or women with a high viral load cannot be made.

\section{Discussion}

Vertical transmission occurs in up to $90 \%$ of double positive $\mathrm{HBsAg} / \mathrm{HBeAg}$ women and $90 \%$ of the perinatally infected infants will develop chronic $\mathrm{HBV}$ infection, progressing to chronic liver disease, cirrhosis and hepatocellular cancer [5-8]. Proper screening of all pregnant women for Hepatitis B Virus (HBV) infection, regardless of risk factors, as stated in $\mathrm{WHO}$ and ECDC guidelines is of paramount importance in our effort to combat perinatal transmission. $\mathrm{HBsAg}(+)$ women with a high viral load should be offered antiviral medication to reduce MTCT transplacentaly, and standing immunoprophylaxis protocols should be implemented to the newborn $[1,2,4,14]$. In Greece, a national prevention program for Hepatitis B with universal screening of pregnant women and vaccination of infants against $\mathrm{HBV}$ is in effect since January 1998.

Seroprevalence of $\mathrm{HbsAg}(+)$ in Greece for the general population has been previously studied in a limited number of studies, ranging from $2.33 \%$ (CI 1.54-3.11) to $3.3 \%$ (CI 2.2-4.7), placing Greece in the intermediate endimicity category [14-16]. Exact data on the seroprevalence of native Greeks is lacking. The fact that $84 \%$ of the migrants living in Greece originate from intermediate or high
Table 1: Number of cases and rate of $\mathrm{HBsAg}(+)$ among pregnant women according to ethnic origin.

\begin{tabular}{|l|c|c|}
\hline \multirow{2}{*}{ Greek } & \multicolumn{2}{|c|}{ HBsAg (+) pregnant women } \\
\cline { 2 - 3 } & $(+) /$ total & Rate $\%(95 \%$ Cl) \\
\hline Greek Roma & $7 / 1417$ & $0.5(0.2-1.1)$ \\
\hline Albanian & $5 / 45$ & $11.1(4.8-23.5)$ \\
\hline Bulgarian & $8 / 188$ & $4.3(2-8.5)$ \\
\hline Romanian & $4 / 70$ & $5.7(2.2-14)$ \\
\hline East Europe & $1 / 36$ & $2.8(0.5-14)$ \\
\hline Far East & $0 / 38$ & $0(0-9.2)$ \\
\hline West Europe & $3 / 8$ & $37.5(10-74)$ \\
\hline Refugees & $0 / 13$ & $0(0-23)$ \\
\hline Total & $0 / 10$ & $0(0-28)$ \\
\hline
\end{tabular}

endimicity countries, definitely poses a threat to the increase of the total number of people living with chronic hepatitis B in Greece [14].

In our study, the mean seroprevalence of pregnant women for HBsAg (+) was $1.5 \%$ (95\% CI 1-2.2). In a study by Papaevangelou et al, that took place during a two-week study period in 2003, 2.89\% of women were $\mathrm{HbsAg}(+)$ [9]. Elefsiniotis et al, estimated the seroprevalence of HBsAg in 26,746 women at reproductive age in Greece from 2003-2005 and found that 1.53\% of women were HBsAg positive [17].

The results are similar in a more recent survey by Karatapanis et al, that took place form 2007-2009, where the total HBsAg (+) prevalence is $1,2 \%$ [18].

Native Greek pregnant women showed a low seroprevalence of $0.5 \%$ (95\% CI 0.2-1) in our study, similar to the most recent surveys by Elefsiniotis et al. and Karatapanis et al., reporting $0.57 \%$ and $0.6 \%$ respectively $[7,18]$. The $1.7 \%$ seroprevalence in Greek pregnant women that Papaevangelou et al. reported in the 2003 study probably highlights the constantly falling prevalence of $\mathrm{HBV}$ in the Greek population [9].

In the present study, Albanian pregnant women had a seroprevalence of $4.3 \%$ (95\% CI 2-8.5) for HbsAg (+). Seroprevalence in Albanian pregnant women living in Greece has always been in excess of that reported for Greeks, reflecting the higher burden of HBV in Albania. In a study by Malamitsi-Puchner et al, published in 1996, HbsAg (+) prevalence of $13,4 \%$ was found among Albanian pregnant women [19] and Papaevangelou et al. in 2006 found a prevalence of $9.8 \%$ [9]. More recent studies show a gradual decrease to $4.9-5.4 \%[17,18]$, very similar to our findings. This trend can be explained by the ever-improving hygiene conditions among Albanian families, as well as the increasing percentage of immunization in this population. In the general Albanian population, the seroprevalence of $\mathrm{HBV}$ is $9.5 \%$, which means that Albania remains a country of high endimicity [20]. This is also reflected in ECDC data on migrants in Europe, where Albanian migrants are considered to display a prevalence of 9\% for Chronic Hepatitis B Infection (CHB) [14].

Our study is the first to our knowledge to report the seroprevalence of Bulgarian and Romanian women separately, and 
not as a whole, like former studies. In our study, Bulgarian women had a seroprevalence of $5.7 \%$ (95\% CI 2.2-14), much higher than that reported in a study by Tsankova et al, where the seroprevalence in Bulgaria for pregnant women is estimated at $2.26 \%$, with residence in rural areas and belonging to an ethnic minority important risk factors [21]. This discrepancy could be explained by the fact that most of the Bulgarian immigrants to Greece belong to the Roma population and are not indigenous Bulgarians. In the ECDC Technical Report on the epidemiological assessment of hepatitis B among migrants in Europe, Bulgaria is considered a country of intermediate endimicity of $4.25 \%$ (CI 2.80-5.70) [14]. Similarly, in a study conducted in 2010 in Bulgaria, the prevalence was estimated at $4.8 \%$ in adults over 20 years old [22].

Romanian pregnant women living in Greece had a seroprevalence of $2.8 \%$ (95\% CI $0.5-14)$ for HBsAg (+) in our study. In a study by Popovici et al. in 2016, the prevalence of HBsAg was 5.1\% in pregnant women in Romania, and HBeAg was present in 7.4\% [23]. Romania in general is a country of intermediate endimicity, with a seroprevalence ranging from $4.4-7.6 \%$ in different studies [14,15,24]. Romania is considered the country contributing the largest absolute number of migrants with $\mathrm{CHB}$ in Europe, with prevalence in migrants of 5.5\% [14].

The Roma population constitutes the most important transnational minority in Europe. Low socioeconomic conditions and lack of quality health care deem them a vulnerable population group and predispose them to infections, such as hepatitis B. In the global attempt to eliminate viral hepatitis, Roma should be considered a high priority group for screening and treatment. In a study conducted in Slovakia in 2011, Roma population had a significantly higher prevalence of HBsAg compared to the non-Roma population $(12.4 \%$ vs. $2.8 \%$ ). Furthermore, HBsAg (+) Roma were more commonly HBV DNA positive compared to HBsAg (+) non-Roma population $(94.3 \%$ vs. $70.0 \%$ ) [25].The estimated Roma population in Europe is 5.2 million, with the vast majority living in Central and Eastern Europe. The Roma population in Greece estimated at 120,000-300,000, although Greek in nationality often behaves as a separate group with discrete characteristics, often nonadherent to national guidelines and immunization practices [26]. A large proportion does not seek medical advice, so the exact prevalence of $\mathrm{HBV}$, as well as other diseases, is actually unknown. In our study, $11.1 \%$ (95\% CI 4.8-23.5) of Roma women were HBsAg (+), but the overall number of Roma participants $(\mathrm{N}=45)$ is not big enough to extract safe conclusions. Papaevangelou et al, had found a seroprevalence of 3.6\% (95\% CI 0.810.2) among the same group [9], while in a study of hepatitis B among Roma children in Athens, 22\% were identified with evidence of past infection (anti-HBc $(+)$ ), while $4 \%$ were chronic carriers (HBsAg (+)) [27]. In the same study by Michos et al. on the HBV vaccination coverage in Roma children, only $13.6 \%$ had evidence of HBV vaccination, compared to $95.9 \%$ of non-Roma children, highlighting the immunization gap in this group [27].

The highest seroprevalence of HbsAg (+) was in women of Far East origin (37.5\%, 95\% CI 10-74). Elefsiniotis et al. reported a seroprevalence of $5.57 \%$ in 251 pregnant women in Greece, being the highest in his study too [17]. In China, HBV infection in women of childbearing age $>20$ years old has a prevalence of $5.64 \%$ of the HBsAg
(+) women, $67.07 \%$ were $\mathrm{HBeAg}(+)$ and $81.93 \%$ were HBVDNA positive [28]. The Chinese migrant population in Europe consist the second largest contributing chronic HBV carrier group among migrants, after Romanians, with an estimated 104,000 people and a seroprevalence of $9.4 \%$ (CI 7.9-11.1) in first generation migrants, compared to $4.3 \%$ (CI 1.9-8.3) in second generation migrants [14]. The extremely high rate found in our study, is definitely misleading and due to the very small size of the sample, but nonetheless, women from China should be treated as high risk and every effort should be made to screen them prenatally.

There were no HbsAg (+) women from Central and Northern Europe, as was the case in the study by Elefsiniotis et al. [17]. This comes of no surprise, since central and northern European countries, such as Austria, Belgium, Germany and Finland have a seroprevalence of $0.2-0.7 \%$ for HBsAg $(+)$ in the general population [14]; taking into account that more than $50 \%$ of cases are attributed to migrant groups, one can easily conclude that the indigenous population will have a very low prevalence. There were no women from Russia or the former Soviet Union (FSU) that were HBsAg (+), probably due to the small sample size, since the seroprevalence of migrants in Europe from the former USSR is $4.7 \%$ and of those from Russia $10.3 \%$ [14]. Nonetheless, migrants from these countries should be considered as high-risk groups. Finally, no refugees from Morocco or Egypt were found to be HBsAg (+), which would be expected since these migrant groups exhibit a low seroprevalence of $0.3 \%$ and $1.1 \%$ respectively in Europe [14]. Syria on the contrary is a country of intermediate-tohigh endimicity with a seroprevalence of 5.62\% [14], but the minute number of Syria refugees in our study, justifies that no cases were detected.

It is evident that compliance to screening guidelines is extremely important in preventing vertical transmission and ECDC points out the importance of screening a minimum of $90 \%$ of pregnant women. Underprivileged groups though, often do not seek medical advice during pregnancy. In a study by Papaevangelou et al., that took place in Athens, prenatal screening for HBV had been undertaken in $91.3 \%$ of the women, much improved than in another study by the same author, where only $63.1 \%$ had been tested $[9,29]$. What was of most interest however is that in two different studies, women that escaped prenatal testing, had a much higher prevalence; in total $5.3 \%$, Greeks 2.8\%, Albanians 7.4\% and Roma 4.3\% [9,18]. These findings emphasize that specific populations should be targeted for prenatal testing and vaccination coverage, as WHO strategy also underlines [1]. Our study did not record the percentage of women with regular prenatal follow-up versus those who only sought medical advice at term, so conclusions on this topic cannot be made.

Important conclusions as for the importance of immunization against hepatitis $B$ in the general population can also be drawn from the data related to seropositivity according to different age groups. In our study, HBsAg (+) pregnant women were almost twice as likely to belong to the 30-39 years old group, as compared to the 20-29 years old group, while there was no case recorded under 20 years, even in women of high-endimicity origin. Popovici et al. show similar results from a study in Romania in 2013, where the seroprevalence of HBsAg $(+)$ of people in the general population over 20 years old was $4.02 \%$ compared to only $1.37 \%$ of those younger than 20 years old [30]. 
Accordingly, in a study by Kevorkyan et al. in Bulgaria in 2010, the HBsAg seroprevalence of $1 \%$ was significantly lower in individuals under 19 years old targeted by immunization, related to the rate of $4.8 \%$ in adults over 20 years of age $(\mathrm{P}<0.05)$ [31]

A limitation of our study is that medical records reviewed did not provide any information either on HBeAg or on HBV viral load. The Society of Maternal and Fetal Medicine (SMFM) of Greece, in accordance with WHO and ECDC, recommend that pregnant women with $\mathrm{HBV}$ infection should have their $\mathrm{HBeAg}$ status and $\mathrm{HBV}$ viral load checked, preferably before the third trimester [7]. In the study by Elefsiniotis et al., only $2.67 \%$ of HBsAg $(+)$ pregnant women in Greece were $\mathrm{HBeAg}(+)$ and from the women whose blood was tested for viral load, only $12.7 \%$ exhibited extremely high serum HBV-DNA levels of $>10^{7}$ copies $/ \mathrm{ml}$, suggesting that only a slight proportion of HBsAg positive women in Greece pose a great risk for vertical transmission to their offspring [17]. Accordingly, in HEPNET study in Greece, an ongoing nationwide retrospective-prospective study initiated in 1997, it was shown that the prevalent form of hepatitis B among adult native Greek patients was HBeAg negative (92.13\%), in comparison with immigrants who were positive in $16.6 \%$ of the cases and had a higher DNA load [32]. Malamitsi et al. reported an HBeAg positivity of 7.5\% in Albanians living in Greece [19]. Despite HBeAg (+) prevalence being rather low in Greece, gynecologists and gastroenterologists should cooperate in diagnosing all HBsAg (+) pregnant women and treating as needed, in the fight towards elimination of MTCT hepatitis B disease.

One of the weaknesses of this study was that the population studied was pregnant women giving birth at one of the three major public hospitals in Crete. Women who gave birth at the private hospitals, might have slightly different seroprevalence rates, as they tend to belong to a higher income category as a whole, and would thus be expected to have better living standards. Additionally, public hospitals tend to have an overrepresentation of minority groups, such as immigrants and Roma, which could adversely affect the mean seroprevalence estimated.

Data was not collected concerning the mode of infection of the women, or information related to high-risk categories for HBV infection was not recorded, i.e. drug abuse or multiple sex partners or if the foreign women were first or second-generation migrants in Greece.

In the future, a prospective study could be implemented, for newborns born to mothers with $\mathrm{HBV}$, which would yield interesting data on the final prevalence of MTCT in Greece and the breaches in prenatal and postnatal treatment, when implemented.

\section{Conclusion}

Screening of pregnant women for HBV is of paramount importance, if the goal set by WHO of $90 \%$ reduction in incidence by 2030 is to be achieved. Knowledge of the HBsAg and HBeAg status allows for timely treatment of pregnant women with nucleoside analogues, if applicable, and immunoprophylaxis of the neonate immediately after birth. Defining specific population groups at risk in each country is fundamental, since MTCT is the principal mode of transmission in settings with high prevalence. This study aimed at revealing the seroprevalence of HBsAg in pregnant women in Crete, and similarly in Greece. Native Greek pregnant women were found to have a low seroprevalence of $0.5 \%$, while certain migrant groups and Roma women exhibited statistically significant higher seroprevalence rates and accounted for $75 \%$ of HBV cases. Taking into account that it is these specific groups that often lack quality health care and are nonadherent to national guidelines and immunizations practices, one can easily perceive the threat to public health. Improvements in perinatal care should aim at timely diagnosing seropositive pregnant women and implicating gastroenterologists in their care. Immunization strategies reaching out to all population groups living in underprivileged conditions is also of paramount importance, since intrafamilial transmission remains a threat and perpetuates the pool of HBsAg (+) future mothers.

\section{Sponsoring}

This study was done without sponsoring from any source.

\section{Approvals}

The ethics committee of each participating hospital approved this study, and the directors of each obstetrics clinic had consented, prior to the beginning of the study.

\section{Confidentiality}

Personal data gathered from all the participating women were reviewed only by the main researcher and were in no way accessible by others.

\section{Acknowledgment}

I would like to thank Professor of Gynecology and Obstetrics of the University of Crete and Director of the Gynecology and Obstetrics Clinic of the University Hospital of Crete, A Makrygiannakis, Professor of Gynecology and Obstetrics of the University of Crete and Director of the Gynecology and Obstetrics Clinic of the Venizeleio General Hospital of Heraklion, I Matalliotakis and G Daskalakis, Director of the Gynecology and Obstetrics Clinic of the General Hospital of Chania for their approval and help in gathering data from the patients' files.

\section{References}

1. WHO. Global health sector strategy on viral hepatitis 2016-2021. Geneva: World Health Organization. 2016.

2. World Health Organization. Regional Office for Europe. Action plan for the health sector response to viral hepatitis in the WHO European Region. Copenhagen, Denmark. 2017

3. WHO Global hepatitis report. Executive summary. 2017.

4. American Academy of Pediatrics. Hepatitis B In: Red Book: 2015 Report of the Committee on Infectious Diseases $30^{\text {th }}$ Edition. Editors: Kimberlin DW Brady MT, Jackson MA, Long SS. Elk Grove Village. American Academy of Pediatrics, 2015: 400-423.

5. Ma L, Alla NR, Li X, Mynbaev OA, Shi Z. Mother-to-child transmission of HBV review of current clinical management and prevention strategies. Rev. Med. Virol. 2014; 24: 396-406.

6. Yi P, Chen R, Huang Y, Zhou RR, Fan XG. Management of mother-to-child transmission of hepatitis $B$ virus: Propositions and challenges. Journal of Clinical Virology. 2016; 77: 32-39.

7. Society for Maternal-Fetal Medicine (SMFM), Dionne-Odom J, Tita AT, Silverman NS. \#38: Hepatitis B in pregnancy screening, treatment, and prevention of vertical transmission. Am J Obstet Gynecol. 2016; 214: 6-14. 
8. Yi P, Chen R, Huang $Y$, Zhou RR, Fan XG. Management of mother-to-child transmission of hepatitis $B$ virus: Propositions and challenges. Journal of Clinical Virology. 2016; 77: 32-39.

9. Papaevangelou V, Hadjichristodoulou C, Cassimos D, Theodoridou M. Adherence to the screening program for HBV infection in pregnant women delivering in Greece. BMC Infectious Diseases. 2006; 6: 84

10. Wen WH, Chang MH, Zhao LL, Ni YH, Hsu HY, Wu JF, et al. Mother-to-infant transmission of hepatitis $B$ virus infection: significance of maternal viral load and strategies for intervention. J Hepatol. 2013; 59: 24-30.

11. Funk AL, Lu Y, Yoshida K, Zhao T, Boucheron P, van Holten J, et al. Efficacy and safety of antiviral prophylaxis during pregnancy to prevent mother-tochild transmission of hepatitis B virus: a systematic review and meta-analysis. Lancet Infect Dis. 2021; 21: 70-84.

12. Prevention of Mother-to-Child Transmission of Hepatitis B Virus: Guidelines on Antiviral Prophylaxis in Pregnancy. Geneva: World Health Organization.

13. Neu N, Duchon J, Zachariah P. TORCH infections. Clinics in Perinatology. 2015; 42: 77-103.

14. ECDC Technical Report: Epidemiological Assessment of Hepatitis B and C among migrants in the EU/EEA. 2016.

15. Hofstraat SHI, Falla AM, Duffell EF, Hahné SJM, Amato-Gauci AJ, Veldhuijzen IK, et al. Current prevalence of chronic hepatitis B and C virus infection in the general population, blood donors and pregnant women in the EU/EEA: a systematic review. Epidemiol Infect. 2017; 145: 2873-2885

16. Drositis I, Bertsias A, Lionis C, Kouroumalis E. Epidemiology and molecular analysis of hepatitis $A, B$ and $C$ in a semi-urban and rural area of Crete. European Journal of Internal Medicine. 2013; 24: 839-845.

17. Elefsiniotis IS, Glynou I, Brokalaki H, Magaziotou I, Pantazis KD, Fotiou A et al. Serological and virological profile of chronic HBV infected women at reproductive age in Greece. European Journal of Obstetrics and Gynecology and Reproductive Biology. 2007; 132: 200-203.

18. Karatapanis S, Skorda L, Marinopoulos S, Papastergiou V, Drogosi M, Lisgos $P$, et al. Higher rates of chronic hepatitis $B$ infection and low vaccinationinduced protection rates among parturient escaping $\mathrm{HBsAg}$ prenatal testing in Greece: a 2-year prospective study. European Journal of Gastroenterology and Hepatology. 2012; 24: 878-883.

19. Malamitsi-Puchner A, Papacharitonos S, Sotos D, Tzala L, Psichogiou M Hatzakis A, et al. Prevalence study of different hepatitis markers among pregnant Albanian refugees in Greece. European Journal of Epidemiology. 1996; 12: 297-301.

20. Resuli B, Prifti S, Kraja B, Nurka T, Basho M, Sadiku E. Epidemiology of hepatitis B virus infection in Albania. World Journal of Gastroenterology. 2009; 15: 849-852.
21. Tsankova GS, Kostadinova T, Todorova TT. Seroprevalence of hepatitis B among pregnant women in Varna Region, Bulgaria. Journal of Medical Virology. 2016; 88: 2012-2015.

22. Kevorkyan A, Teoharov P, Lernout T, Petrova N, Raycheva R, Ivanov I, et al. Prevalence of HBV and HCV among outpatients in the Plovdiv region of Bulgaria, 2010-2011. J Med Virol. 2015; 87: 401-406.

23. Popovici O, Radu R, Romaniuc A, Azoicăi D. A seroprevalence study for hepatitis $B$ virus markers of infection in pregnant women in Romania: results and opportunities for prevention. J Gastrointestin Liver Dis. 2018; 27: 133137.

24. Gheorghe L, Csiki IE, lacob S, Gheorghe C. The prevalence and risk factors of hepatitis B virus infection in an adult population in Romania: a nationwide survey. European Journal of Gastroenterology and Hepatology. 2013; 25: $56-64$.

25. Drazilova S, Janicko M, Kristian P, Schreter I, Halanova M, Urbancikova I, et al, HepaMeta Team. Prevalence and Risk Factors for Hepatitis B Virus Infection in Roma and Non-Roma People in Slovakia. Int J Environ Res Public Health. 2018; 15: 1047

26. Mrzljak A, Bajkovec L, Vilibic-Cavlek T. Hepatotropic viruses: Is Roma population at risk? World J Gastroenterol. 2021; 27: 143-151.

27. Michos A, Terzidis A, Kalampoki V, Pantelakis K, Spanos T, Petridou ET Seroprevalence and risk factors for hepatitis A, B and C among Roma and non-Roma children in a deprived area of Athens, Greece. Journal of Medical Virology. 2008; 80: 791-797

28. Ding $\mathrm{Y}$, Sheng Q, Ma L, Dou X. Chronic HBV infection among pregnant women and their infants in Shenyang, China. Virol J. 2013; 10: 17.

29. Papaevangelou G, Farmaki G, Kada H. Hepatitis B maternal-fetal transmission in Southern Europe. Intervirology. 1998; 41: 197-200.

30. Popovici O, Molnar GB, Popovici F, Janţă D, Pistol A, Azoicăi D. A Seroprevalence Study of Hepatitis B and C Virus Infections in a Hospitalized Population in Romania, an Opportunity for a Better National Prevention and Control Strategy. J Gastrointestin Liver Dis. 2016; 25: 25-32.

31. Kevorkyan A, Teoharov P, Lernout T, Petrova N, Raycheva R, Ivanov I, et al. Prevalence of HBV and HCV among outpatients in the Plovdiv region of Bulgaria, 2010-2011. J Med Virol. 2015; 87: 401-406.

32. Raptopoulou-Gigi M, Orphanou E, Lalla TH, Lita A, Garifallos A. Prevalence of hepatitis $\mathrm{C}$ virus infection in a cohort of pregnant women in northern Greece and transmission of HCV from mother to child. European Journal of Epidemiology. 2001; 17: 263-266 